\title{
Primary Primitive Neuroectodermal Tumor of the Breast: a Case Report
}

\author{
Kyungran Ko, MD' \\ Eun Ah Kim, MD ${ }^{1}$ \\ Eun Sook Lee, MD² \\ Youngmee Kwon, MD
}

\begin{abstract}
Primary primitive neuroectodermal tumors (PNET) are rare malignant tumors, affecting mostly children and adolescents. Only three cases of primary breast PNETs have been reported in the medical literature, with none in Korea. We present a case of a primary PNET of the breast in a 33-year-old woman, with imaging and immunohistopathology findings.
\end{abstract}

Index terms:

Primitive neuroectodermal tumor (PNET)

Breast

Sarcoma

DOI:10.3348/kjr.2009.10.4.407

Korean J Radiol 2009; $10: 407-410$

Received November 3, 2008; accepted after revision November 24, 2008.

Departments of 'Radiology and ${ }^{3}$ Pathology, Hospital and Research Institute, National Cancer Center, Goyang-city 410-769, Korea; ' 2 epartment of Surgery, Medical College, Korea University, Seoul 136-705, Korea

Address reprint requests to: Eun Sook Lee, MD, Department of Surgery, Medical College, Korea University, 126-1 Anam-dong $5 \mathrm{Ga}$, Seongbuk-gu, Seoul 136-705, Korea. Tel. (822) 920-6744

Fax. (822) 920-6743

e-mail: eslee@korea.ac.kr
$\mathrm{P}$ rimitive neuroectodermal tumors (PNET) are rare, malignant, smallround-cell tumors of the bone and soft tissue that usually occur in children and young adults (1). In adults, they are extremely rare, but have been reported in the chest wall and other body parts, including the breast (2-4). Here, we present a case of a primary PNET of the breast in a 33-year-old woman, with a brief review of the current literature.

\section{CASE REPORT}

A 33-year-old woman was referred to the Center for Breast Cancer because of a growing, palpable mass in her left breast. Two years earlier, she had an excisional biopsy for fibroadenoma in her left breast at another hospital. During her follow-up care, new lesions were found in her left breast, and these lesions grew over a 6-month period. One of the masses was biopsied using a Mammotome ${ }^{\circledR}$ (Johnson \& Johnson, New Brunswick) biopsy device under sonographic guidance. The initial pathologic diagnosis was a PNET.

After referral to our hospital, the patient underwent positron emission tomography (PET) and no uptake was reported. Bilateral mammography was performed and showed an ill-defined, isodense mass deep in the left upper inner quadrant, without evidence of microcalcifications (Fig. 1A). Sonography revealed two adjacent circumscribed, oval, homogeneously low echoic masses with posterior acoustic enhancement (Fig. 1B). A flow signal was not seen on color Doppler sonography. Magnetic resonance imaging (MRI) revealed two adjacent circumscribed masses with intermediate signal intensity on T1-weighted images and high signal intensity on T2-weighted images. After gadolinium administration, intense enhancement with washout was seen (Fig. 1C). There was no evidence of infiltration into the chest wall. The patient underwent a left lumpectomy.

The lumpectomy specimen of the left breast revealed a relatively well-circumscribed tumor, measuring $2.5 \times 2.0 \times 1.5 \mathrm{~cm}$. The cut surface of the tumor was grayish tan, fish flesh-like, and slightly friable (Fig. 1D). Microscopically, the tumor was composed of small, round cells with inconspicuous nucleoli and scanty cytoplasm. The tumor cells 
were arranged in diffuse or compact sheets or lobules (Fig. 1E). By immunohistochemical staining, the tumor cells were strongly positive for vimentin, CD99 (Fig. 1F), and FLI-1 but were negative for cytokeratin, leukocyte common antigen, synaptophysin, chromogranin, CD56, desmin, and myoD1.

Six months after the lumpectomy, the patient had no evidence of metastasis or local recurrence.

\section{DISCUSSION}

Primitive neuroectodermal tumors are uncommon, malignant, small-round-cell tumors that arises in soft tissues or bone, most commonly in children and young adolescents. Primary PNETs demonstrate a predilection for the truncal and axial soft tissue, including the chest wall (Askin tumor), the paravertebral region (50-60\% of cases), and the extremities (20-25\% of cases) (5). The thoracopulmonary region (Askin tumor) is the single most common primary site. Primary PNETs of many organs of the body have been documented (6-11), but only three papers report primary PNET of the breast (2-4).

Previous reports of primary breast PNET show common clinical findings of a growing mass, over a 2-year or 4- month follow-up $(2,3)$. In our case, the mass grew over a 6-month period. PNETs of other visceral organs usually present with a painful mass and constitutional symptoms (5), but in this case, the patient only complained of a palpable, growing mass.

Maxwell et al. (3) described sonographic findings of primary PNET of the breast as a superficial, circumscribed, hypoechoic mass with posterior acoustic enhancement and an apparent hypoechoic tract extending to the skin. These lesions were misdiagnosed as epidermal inclusion cysts and considered benign (3). In our case, sonographic findings were similar, but the mass was deep and the mammogram showed no microcalcifications.

Our case was an adult patient with small PNETs on her left breast and no other lesions, such as in the thoracopulmonary region, bone, or other organs, suggesting that the breast is the primary site. Immunohistochemistry and histology were necessary to confirm the diagnosis. In our case, the tumor was composed of small, round cells with inconspicuous nucleoli and scanty cytoplasm. The tumor cells were arranged in diffuse or compact sheets or lobules (Fig. 1E). By immunohistochemical staining, the tumor cells were strongly positive for vimentin, CD99 (Fig. 1F), and FLI-1. CD99 (MIC2) is a cell surface glycoprotein

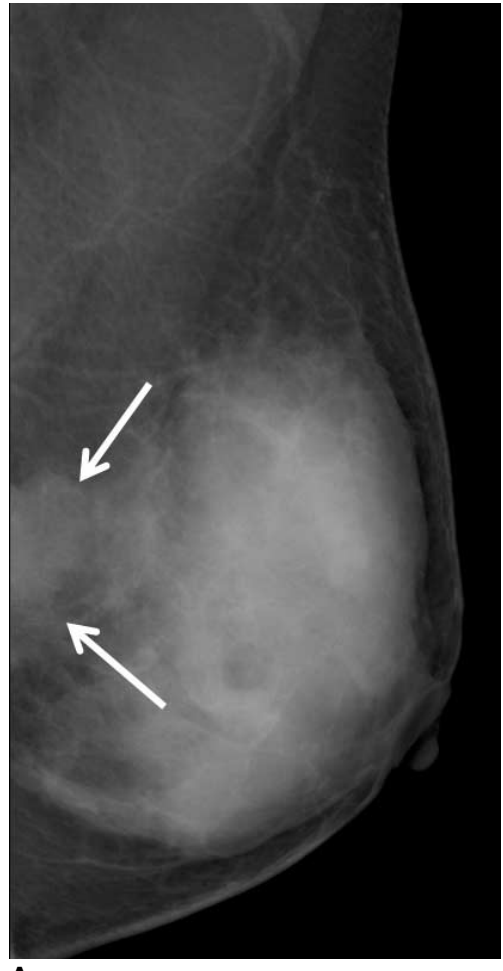

A

Fig. 1. Primary primitive neuroectodermal tumors in 33-year-old woman with palpable mass in left breast.

A. Mediolateral oblique mammogram of left breast shows ill-defined, isodense mass deep in upper inner quadrant (arrows).

B. Sonography reveals two adjacent, circumscribed, oval, homogeneously hypoechoic masses with posterior acoustic enhancement.

C. On breast MRI, mass showed intermediate signal intensity on T1-weighted image (T1) and high signal intensity on T2-weighted image (T2). Intense enhancement was seen during contrast enhancement (CE) (arrows). 

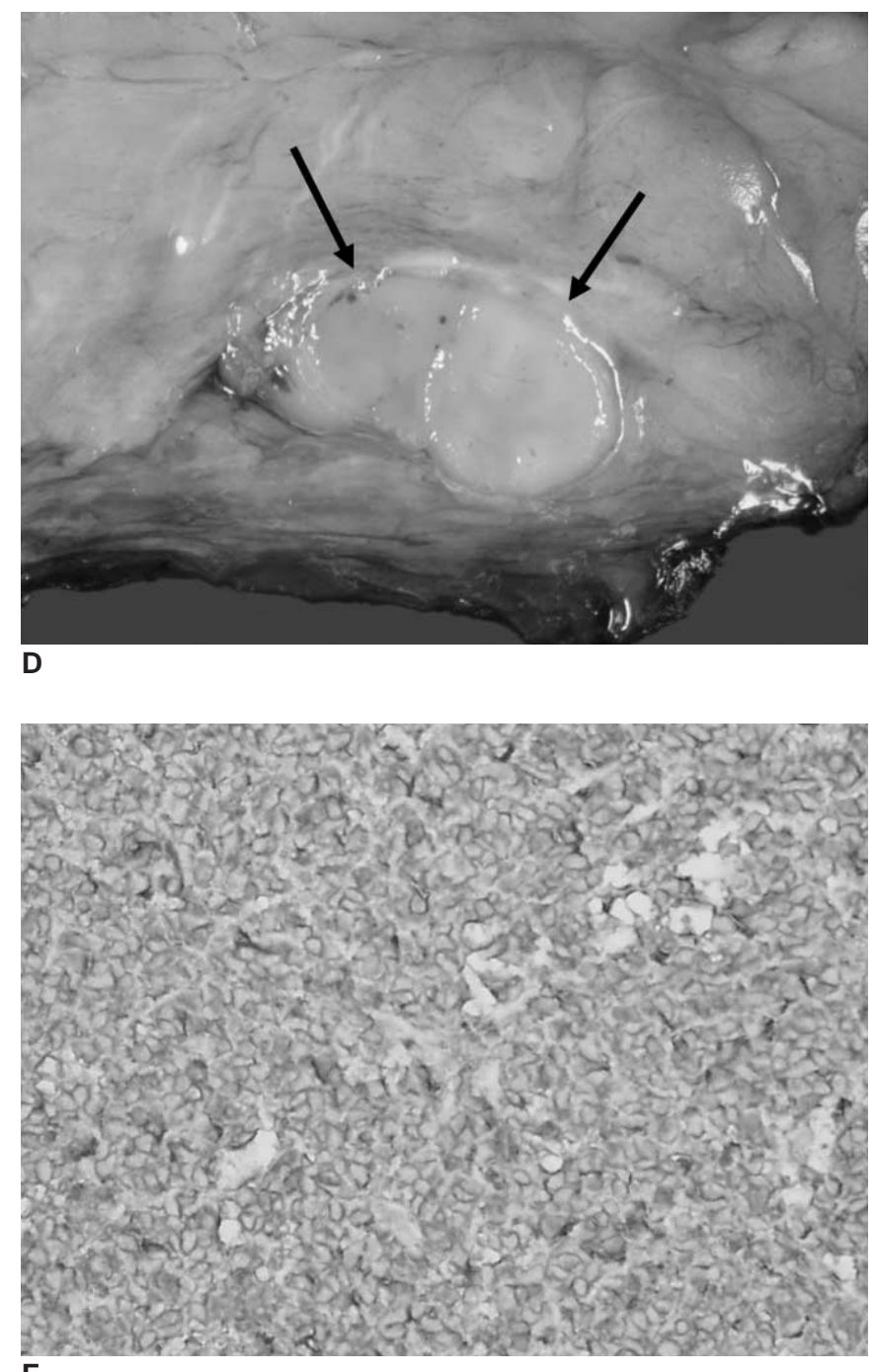

$\mathbf{F}$

involved in cell adhesion and CD99 positivity confirms the diagnosis of PNET $(2,4)$. In addition, fluorescence in situ hybridization is highly specific for Ewing's sarcoma and PNET (4).

The findings in computed tomography (CT) and MRI of PNETs cannot differentiate PNETs from other types of bone and soft tissue tumors $(5,12)$, but they usually appear as large, non-calcified, soft tissue masses with a heterogeneous appearance and cystic or necrotic areas on CT. By MRI, signal intensity was similar to muscle on T1weighted images and heterogeneously high on T2weighted images, with variable gadolinium enhancement. Bright, heterogeneously high signal intensity on T2weighted images is caused by focal areas of hemorrhage or necrosis (12). We obtained MR images with gadolinium enhancement, which showed a washout pattern.

In conclusion, we diagnosed a primary breast PNET. Although primary breast PNET is extremely rare, a PNET

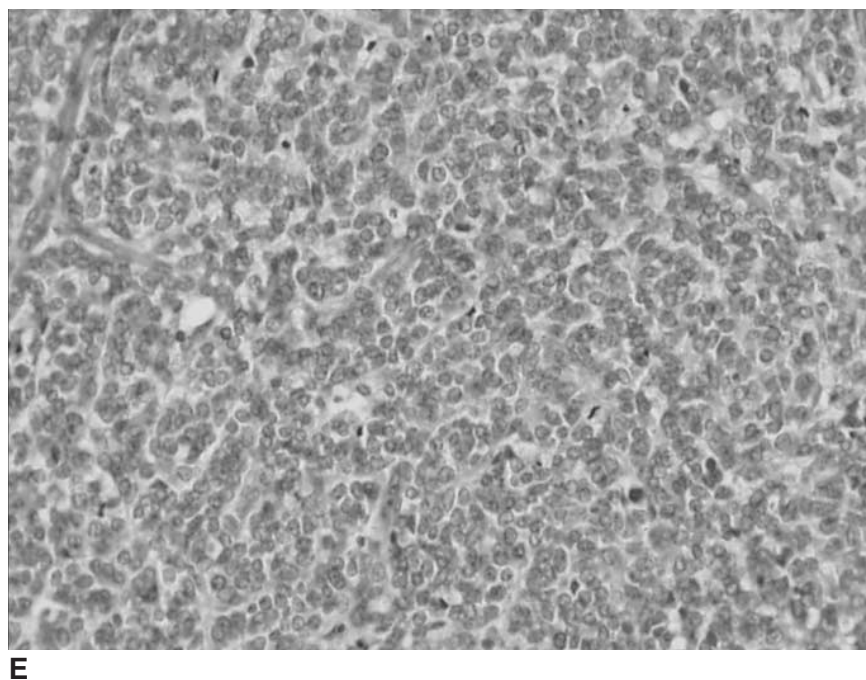

Fig. 1. Primary primitive neuroectodermal tumors in 33-year-old woman with palpable mass in left breast.

D. Grossly, tumor is well-circumscribed, grayish tan, fish flesh, and slightly friable (black arrows).

E. Microscopically, tumor is composed of small, round cells with inconspicuous nucleoli and scanty cytoplasm, which are arranged in sheets or solid nests (Hematoxylin \& Eosin staining, $\times 200$ ).

F. On immunohistochemical staining, tumor cells show strong membranous immunoreactivity for CD99.

differential diagnosis might be considered with a growing, morphologically benign mass.

\section{References}

1. Tefft M, Vawter GF, Mitus A. Paravertebral "round cell" tumors in children. Radiology 1969;92:1501-1509

2. da Silva BB, Lopes-Costa PV, Pires CG, Borges RS, da Silva RG Jr. Primitive neuroectodermal tumor of the breast. Eur J Obstet Gynecol Reprod Biol 2008;137:248-249

3. Maxwell RW, Ghate SV, Bentley RC, Soo MS. Primary primitive neuroectodermal tumor of the breast. J Ultrasound Med 2006;25:1331-1333

4. Tamura G, Sasou S, Kudoh S, Kikuchi J, Ishikawa A, Tsuchiya $\mathrm{T}$, et al. Primitive neuroectodermal tumor of the breast: immunohistochemistry and fluorescence in situ hybridization. Pathol Int 2007;57:509-512

5. Ibarburen C, Haberman JJ, Zerhouni EA. Peripheral primitive neuroectodermal tumors. CT and MRI evaluation. Eur J Radiol 1996;21:225-232

6. Chung $\mathrm{CH}$, Wang $\mathrm{CH}$, Wang TY, Huang JK, Leu YS. Extraskeletal Ewing sarcoma mimicking a thyroid nodule. 


\section{Ko et al.}

Thyroid 2006;16:1065-1066

7. Danner DB, Hruban RH, Pitt HA, Hayashi R, Griffin CA, Perlman EJ. Primitive neuroectodermal tumor arising in the pancreas. Mod Pathol 1994;7:200-204

8. Hart MN, Earle KM. Primitive neuroectodermal tumors of the brain in children. Cancer 1973;32:890-897

9. Jimenez RE, Folpe AL, Lapham RL, Ro JY, O'Shea PA, Weiss SW, et al. Primary Ewing's sarcoma/primitive neuroectodermal tumor of the kidney: a clinicopathologic and immunohistochemical analysis of 11 cases. Am J Surg Pathol 2002;26:320-327
10. Kang MS, Yoon HK, Choi JB, Eum JW. Extraskeletal Ewing's sarcoma of the hard palate. J Korean Med Sci 2005;20:687-690

11. Lee YY, Kim do H, Lee JH, Choi JS, In KH, Oh YW, et al. Primary pulmonary Ewing's sarcoma/primitive neuroectodermal tumor in a 67-year-old man. J Korean Med Sci 2007;22:S159S163

12. Winer-Muram HT, Kauffman WM, Gronemeyer SA, Jennings SG. Primitive neuroectodermal tumors of the chest wall (Askin tumors): CT and MR findings. AJR Am J Roentgenol 1993;161:265-268 